\title{
Las Reuniones Consultivas del Tratado Antártico: Desafíos del consenso ante los nuevos escenarios de negociación ${ }^{1}$
}

\section{Antarctic Treaty Consultative Meetings: Challenges to consensus in front of the new negotiation scenarios}

\author{
Chantal Lazen Muñoz \\ chantal.lazen@hotmail.com \\ Universidad de Chile
}

Resumen: La regla del consenso ha sido útil para dar estabilidad a la política antártica. Sin embargo, la evolución de este régimen internacional plantea nuevos escenarios de negociación, en que ella pudiera resultar insuficiente. Se analizan los principales desafíos que enfrentan en este sentido las Reuniones Consultivas el Tratado Antártico como instancia de toma de decisiones del gobierno del Sexto Continente.

Palabras claves: Sistema del Tratado Antártico - Reuniones Consultivas del Tratado Antártico consenso

\begin{abstract}
The consensus rule has been useful to guarantee the stability of the Antarctic politics. However, this international regimen's evolution creates new scenarios of negotiation where this rule could be insufficient. Main challenges to the Antarctic Treaty Consultative Meetings as a decision-making forum to the Antarctic government are analyzed.
\end{abstract}

Keywords: Antarctic Treaty System - Antarctic Treaty Consultative Meetings - consensus

1 Ponencia presentada en las Jornadas Chilenas de Derecho Antártico 2019, Facultad de Derecho, Universidad de Chile, 28 de marzo del 2019. 
Las Reuniones Consultivas del Tratado Antártico: Desafíos del consenso ante los nuevos escenarios de negociación Chantal Lazen Muñoz

\section{Introducción}

Al adoptarse el Tratado Antártico se estableció, en su artículo IX (1) que, las Partes Contratantes deben reunirse "a intervalos y en lugares apropiados, con el fin de intercambiar informaciones, consultarse mutuamente sobre asuntos de interés común relacionados con la Antártida, y formular, considerar, y recomendar a sus Gobiernos medidas para promover los principios y objetivos del presente Tratado".

Las Reuniones Consultivas del Tratado Antártico (RCTA) son el medio a través del cual se pone en práctica lo señalado en este artículo, en tanto se constituye como un foro internacional que convoca a las Partes Contratantes y a otros miembros del Sistema del Tratado Antártico (STA) a discutir temas de relevancia relacionados con la gobernanza y administración de la Antártica, con el fin de que, a través del consenso, se tomen decisiones en base a lo discutido.

Estas reuniones anuales son el símil del Poder Legislativo en la Antártica. Se le considera el foro internacional de más alto nivel responsable de la protección y gestión de la región antártica (Thorp, 2012). Sin embargo, no es solo un foro regulador sino que también cumple otras funciones.

En este orden de ideas, las Reuniones Consultivas son: (i) un foro político, ya que permiten el diálogo político entre los estados parte en el tratado; (ii) un foro de coordinación, porque a través de estas reuniones se entregan informes de los observadores a los representantes de las partes y de esta manera se puede coordinar el Sistema Antártico (Villamizar, 2013).

El proceso de toma de decisiones en la RCTA se realiza a través del consenso, y solo pueden participar en él las Partes Consultivas del Tratado Antártico. A través de este mecanismo se asegura que quienes regulan y dirigen la Antártica son quienes están realmente interesados en desarrollar investigaciones científicas en el continente.

El criterio de actividades, que vincula la autoridad de toma de decisiones con las actividades reales en la Antártica, es un importante estímulo para la cooperación allí. Las decisiones sobre las actividades en la Antártica son tomadas por aquellos que las llevan a cabo: un incentivo para basar las decisiones en la experiencia común y compartida sobre la Antártica y un elemento disuasivo para politizar las cuestiones. Por otra parte, este criterio tiende a restringir el posible abuso de poder de objeción que podría darse en la toma de decisiones por consenso. Estas disposiciones legales y políticas han sido ingredientes esenciales en el práctico logro de los objetivos que se encuentran en el corazón del Tratado Antártico (Scully, 2011).

Número de página no utilizable para citar 
La RCTA no solo ha crecido en cantidad de miembros, sino que también han cambiado los desafíos y problemáticas que enfrenta y el entorno en el que opera. Es así como hoy en día las RCTA tienen la tarea de adaptarse y hacer frente a nuevos escenarios internacionales de negociación, tales como el incremento en la heterogeneidad de sus miembros y la creciente complejización de sus agendas de trabajo.

\section{Cambio en los escenarios de negociación}

El contexto político en el cual se dio lugar a las negociaciones del tratado es muy distinto al que contemplamos hoy en día. Firmado durante la guerra fría, el Tratado Antártico buscaba conciliar los distintos intereses geoestratégicos y tensiones entre países que hacían reclamaciones territoriales y asegurar la el uso pacífico del mismo a través de la cooperación. A través de los años han ido surgiendo distintos intereses en este continente, pero podemos decir que hoy en día, frente a la amenaza del cambio climático y los recursos limitados, predominan los intereses medioambientales, aunque en el contexto de un mundo globalizado y de economías abiertas, también los intereses comerciales.

A continuación se describirán los principales cambios en los escenarios de negociación del Sistema del Tratado Antártico.

\subsection{Incremento actividad humana}

Esto como un producto de crecientes intereses comerciales, pues la actividad humana ha aumentado significativamente debido a la industria del turismo. De acuerdo al informe presentado por la International Association of Antarctica Tour Operators (IAATO) en la RCTA $2018^{2}$, el turismo marítimo comercial tradicional en la Antártica ha ido en aumento y representa más del 95\% de la actividad en tierra.

En este mismo documento, se da cuenta de que durante la temporada 2017/2018, 42.576 personas (incluidos operadores terrestres de la IAATO) desembarcaron en la Antártica, superando la cifra de la temporada anterior. La IAATO señaló que eso se debía, en parte, a que los barcos operaban con una mayor capacidad de pasajeros y a que la industria se estaba beneficiando de un crecimiento económico sólido en todo el mundo. Adicionalmente, las

2 Panorama de la IAATO sobre el turismo antártico: temporada antártica 2017/2018 y cálculos preliminares para la temporada 2018/2019.

Número de página no utilizable para citar 
Las Reuniones Consultivas del Tratado Antártico: Desafíos del consenso ante los nuevos escenarios de negociación Chantal Lazen Muñoz

estimaciones de la IAATO para la temporada 2018/2019 indicaron que la cantidad de pasajeros aumentaría a alrededor de 55.764 individuos, en línea con la creciente tendencia mundial de viajar a destinos remotos y de altas latitudes. ${ }^{3}$

\subsection{Cambio climático}

Situación de amplio conocimiento debido a su envergadura global, y particularmente relevante en la Antártica debido a la implicancia de este continente en el clima terrestre, ya que la Antártica forma parte necesaria del proceso de redistribución del calor entre el Ecuador y los polos.

En ese sentido, el SCAR ha puesto de relieve algunos estudios que muestran evidencia de que el Océano Austral se está calentando a una velocidad mucho mayor y a mayores profundidades que en otros lugares del mundo, hay una invasión de especies no autóctonas en el ámbito marino, y una disminución de los pingüinos Adelia reproductores, que se asocia a la disminución del hielo marino. ${ }^{4}$ Esto da cuenta de un evidente deterioro del ecosistema y biodiversidad antárticos.

\subsection{Avances tecnológicos}

Este factor implica una complejización de las agendas de trabajo, ya que estas van incorporando nuevos elementos, de alto contenido científico, algunos no regulados en el tratado original o el protocolo, y que requieren atención ante la incertidumbre de los posibles efectos colaterales en su utilización.

Luego de una inspección realizada a siete instalaciones entre el 9 y el 17 de febrero de 2018, Noruega señalo, en el contexto de la RCTA 2018, que existía una tendencia sostenida a adoptar sistemas tecnológicos más complejos que permitirán la operación remota en mayor medida que los sistemas anteriores. Se indicó que esto genera oportunidades nuevas e interesantes en relación con la eficiencia operativa, las operaciones autónomas y la recolección remota de datos, entre otros aspectos. Pero que también podría generar algunos riesgos, ya que las estaciones serían más vulnerables y dependerían de personal especializado, además de estar expuestas a riesgos cibernéticos. $^{5}$

XLI RCTA, Informe Final, párr. 73.

XL RCTA, Informe Final, párr. 334.

5 XLI RCTA, Informe Final, párr. 60.

Número de página no utilizable para citar 


\section{Desafíos de la regla de consenso}

Los principales desafíos relacionados con la regla del consenso son los siguientes:

\subsection{Mayor diversidad entre miembros}

El número de Estados que adhieren al Tratado Antártico van en aumento. Actualmente son 53 Estados que han firmado el tratado y de estos, 29 han alcanzado el estatus consultivo. Es decir, el proceso de negociación y de toma de decisiones se ha complejizado en tanto hay más intereses en pugna de los que habían al darse origen al tratado.

Algunos de los miembros más recientes son Malasia y Pakistán, que en su momento mantuvieron una posición crítica respecto al Sistema del Tratado Antártico.

Este aumento en la diversidad de Estados interesados en el ATS se ve reflejado también en las distintas tradiciones jurídicas presentes. Esto puede complejizar el proceso de toma de decisiones en un ámbito doméstico, en tanto que para que las normas adoptadas en las RCTA sean efectivamente aplicadas, requieren ser implementadas en las legislaciones domesticas de las partes. La diversidad de sistemas jurídicos, así como el gran número de disposiciones dictadas, originan un proceso de recepción normativa complejo y una aplicación práctica muchas veces poco efectiva (Ferrada, 2018).

\subsection{Vacíos legales}

Los casos más paradigmáticos son el turismo y la bioprospección.

En cuanto al turismo, este es un tema en constante discusión en las RCTA. Las partes muestran preocupación ante el aumento del turismo y la diversificación de actividades turísticas, pero aun así no se ha llegado a consenso en cuanto a una regulación integral del fenómeno. Si bien se han acordado Medidas diseñadas para regular y mejorar la gestión del turismo, estas aun no entran en vigor. Es el caso de la Medida 4 (2004), Medida 1 (2005) y Medida 15 (2009) ${ }^{6}$

En la RCTA 2018, Argentina destacó las dificultades que implicaba la presencia de operadores no pertenecientes a la IAATO para la coordinación de operaciones, tanto con respecto a la

6 XLI RCTA, Informe Final, párr. 83.

Número de página no utilizable para citar 
Las Reuniones Consultivas del Tratado Antártico: Desafíos del consenso ante los nuevos escenarios de negociación Chantal Lazen Muñoz

recolección de estadísticas precisas sobre turismo como al cumplimiento de las Medidas de la RCTA relativas al turismo. Indicó también que era necesario que las Partes definieran estrategias claras sobre cómo manejarse con operadores no pertenecientes a la IAATO. Así, el Informe Final señaló que las dificultades significativas en relación con la gestión del turismo seguían pendientes de análisis por parte de la RCTA, lo que incluía el impacto del turismo no autorizado y un mayor crecimiento en las cifras del turismo y los tipos de actividades turísticas.

Este tema se ha incluido en los planes de trabajo estratégicos plurianuales, lo que significa que se considera un tema prioritario. Sin embargo, no implica necesariamente que se acelerará el proceso de toma de decisiones en cuanto a una posible regulación.

Por otro lado, en cuanto a la bioprospección, al igual que sucede con el turismo, se han dado numerosas discusiones, pero sin decisiones conclusivas y se ha pospuesto para otras RCTA. Un argumento recurrente en las discusiones es que se considera necesario adoptar una definición práctica de bioprospección biológica, pero frente a las propuestas, no se ha llegado a consenso en cuanto a una que no sea problemática para una o más partes. Se reconocen las problemáticas de no tener una regulación al respecto y se han realizado informes en cuanto a recolección y uso de organismos biológicos, y posibles dificultades con el registro de patentes, en tanto podría ser contrario al principio de la libre disponibilidad de observaciones científicas establecido en el tratado.

La Antarctic and Southern Ocean Coalition (ASOC) consideró que hacía falta más claridad y transparencia en el modo de desarrollo de las actividades de prospección biológica en la Antártida y en cómo estas afectaban de forma directa o indirecta el medioambiente y otros valores antárticos. La ASOC alentó a las Partes a apoyar nuevos debates sobre el tema y a implementar los requisitos de intercambio de información de la Resolución 7 (2005) y la Resolución 6 (2013). ${ }^{7}$

\subsection{Paralización normativa}

En relación con el punto anterior, las RCTA han cesado de adoptar Medidas vinculantes en temas que no sean sobre Zonas Antárticas especialmente protegidas o Zonas Antárticas especialmente administradas.

Un ejemplo de esto es lo que sucede con el Anexo VI del Protocolo sobre responsabilidad emanada de emergencias ambientales. Este fue adoptado bajo la Medida 1 (2005) y no ha entrado

7 XLI RCTA, Informe Final, párr. 53.

Número de página no utilizable para citar 
en vigor en 13 años, habiéndolo aprobado solo 14 de 28 Estados necesarios para su entrada en vigencia. La discusión se sigue posponiendo.

En la RCTA 2017 se dio cuenta que entre las Partes que han aprobado el Anexo VI (Australia, Ecuador España, Finlandia, Italia, Noruega, Nueva Zelandia, los Países Bajos, Perú, Polonia, el Reino Unido, Sudáfrica, y Suecia), cinco informaron que la aplicación del Anexo VI en su legislación nacional se encontraba a la espera de la entrada en vigor del Anexo VI (la Federación de Rusia, Finlandia, Noruega, los Países Bajos, y Suecia). Otras Partes señalaron que el Anexo VI entraría en vigor en sus legislaciones en el momento de su entrada en vigor. Se alentó a las Partes que aún no han aprobado el Anexo VI a que lo hagan como un asunto prioritario, y se señaló que si bien se alcanzó el punto intermedio (14 de las 28 aprobaciones requeridas) ya han transcurrido 12 años desde la aprobación del Anexo ${ }^{8}$.

\section{Referencias bibliográficas}

FERRADA, Luis Valentín (2018), "Five factors that will decide the future of Antarctica", The Polar Journal, 8(1): 84-109.

SCULLY, Tucker (2011), "The Development of the Antarctic Treaty System”, en Science Diplomacy: Antarctica, Science, and the Governance of International Spaces por Paul Arthur Berkman et al., edit., (Washington, D.C.: Smithsonian Institution Scholarly Press), 29-38.

ThORP, Arabella (2012), "Antarctica: the treaty system and territorial claims", House of Commons Library, https://researchbriefings.parliament.uk/ResearchBriefing/Summary/SN05040, [consulta: 02.07.2018].

Villamizar Lamus, Fernando (2013), "Antarctic Treaty and Antarctic Territory Protection Mechanisms", Revista Chilena de Derecho (Pontificia Universidad Católica de Chile), 40: 461-488.

8 XL RCTA, Informe Final, párr. 155.

Número de página no utilizable para citar 